\title{
Management of Compound Odontoma - A Case Report
}

\author{
S. P. Swathi, E. Rajendra Reddy, M. Kiranmayi, M. P. Srujana \\ Department of Paediatric and Preventive Dentistry, Kamineni Institute of Dental Sciences, Narketpally, Telangana, India \\ E-mail for correspondences: swati4389@gmail.com
}

\begin{abstract}
Odontomas represent hamartomatous proliferations of odontogenic epithelium and ectomesenchyme. Odontomas are classified under benign calcified odontogenic tumors, which are composed of more than one type of tissue (enamel and dentin) with anatomic resemblance to normal tooth (composite compound odontoma) or simply as an irregular mass (complex compound odontoma). The present case report describes a case of compound odontoma in a 15-year-old male child.
\end{abstract}

Key words: Compound odontoma, odontogenic tumor, retained primary teeth, unerupted.

\section{INTRODUCTION}

Odontomas are benign tumors of odontogenic origin combining mesenchymal and epithelial elements. ${ }^{[1]}$ Odontomas constitute about $22 \%$ of all odontogenic tumors. The term odontoma was first coined by Broca (1866). ${ }^{[2]}$ Occurs mostly in males (59\%) when compared to females (41\%) with predilection to anterior maxilla (91\%), and occurs frequently on right side of jaw. ${ }^{[2]}$

According to literature, three types of odontomas have been recognized clinically: Intraosseous (central) odontoma, extraosseous (peripheral) odontoma, and erupted odontoma. Intraosseous odontomas are most commonly located inside the bone and are discovered accidentally or due to aplasia or impaction of a permanent or deciduous tooth. ${ }^{[3]}$

Treatment of choice is surgical removal of tooth-like structures in all cases, followed by the histopathological study to confirm the diagnosis. ${ }^{[4]}$ The present case report is of compound odontoma in

\begin{tabular}{|c|c|}
\hline Quick Response Code & Article Info: \\
\hline & doi: $10.5866 / 2018.10 .10122$ \\
\hline 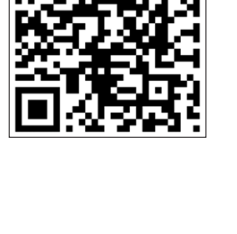 & $\begin{array}{l}\text { Received: } 22-06-2018 \\
\text { Revised: } 26-07-2018 \\
\text { Accepted: } 22-08-2018 \\
\text { Available Online: } 25-09-2018 \text {, (www. } \\
\text { nacd.in)@ NAD, } 2018 \text { - All rights } \\
\text { reserved }\end{array}$ \\
\hline
\end{tabular}

a 15-year-old male that has caused delayed eruption of the right permanent central incisor.

\section{CASE REPORT}

A 15-year-old male patient reported to the Department of Pediatric Dentistry, Kamineni Institute of Dental Sciences, with the chief complaint of unerupted permanent tooth in upper front tooth region. Intraoral examination revealed retained primary anterior and posterior teeth in both the jaws. On soft tissue examination, a firm soft tissue bulge (approximately $2-3 \mathrm{~mm}$ ) in upper anterior region i.r.t to 51 was observed. Based on clinical findings and to assess the status of unerupted permanent dentition, further, investigations such as orthopantomogram and Intra oral periapical radiograph (IOPA) were advised.

IOPA revealed a well-defined irregular radiopaque mass $(0.5 \mathrm{~mm} 0.5 \mathrm{~mm})$ which had similar density of dental tissue [Figure 1]. The radiopaque mass was present apical to the right primary central incisor (51) and coronal to permanent central incisor (11), thereby causing hindrance for the eruption of the right permanent central incisor. OPG depicted the presence of retained deciduous teeth in both upper and lower jaws with succedaneous permanent teeth and radiopaque teeth-like structures (51 and 21) [Figure 2]. As the irregular radiopaque mass was positioned within the bone (intraosseous), extraction of 51 and surgical removal of the tooth-like structures was planned. 


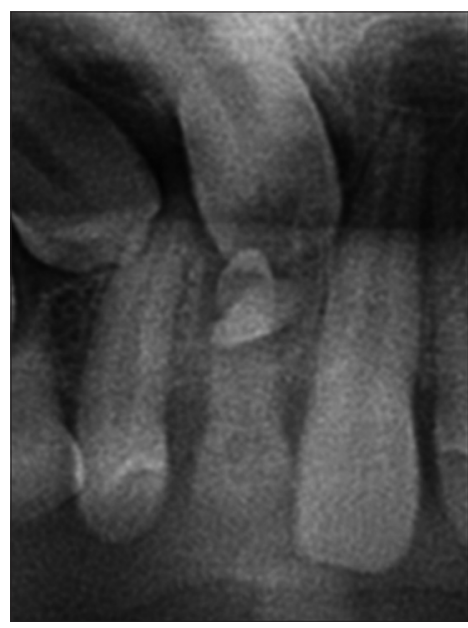

Figure 1: IOPA reveals a well-defined irregular radiopaque mass measuring about $0.5 \mathrm{~mm} \times 0.5 \mathrm{~mm}$ in size and has similar density of dental tissue

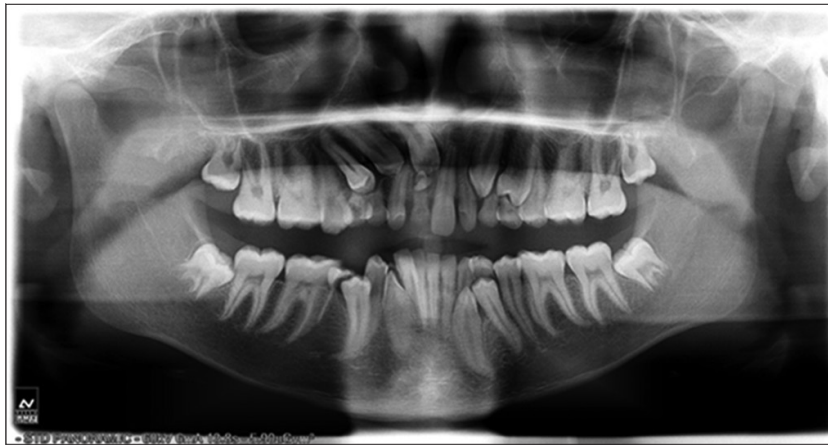

Figure 2: OPG shows the presence of retained deciduous teeth in both upper and lower jaws with succedaneous permanent teeth and radiopaque teeth-like structures irt 51 and 21

\section{Management}

After administration of local anesthesia, initially, extraction of 51 was carried out. Later, a crevicular incision was given to reflect the thick mucoperiosteal flap. Since bone covering was thin, there was no need for cutting bone. The thin superficial layer of bone was removed using curette. Teeth-like small structures were then removed using periosteal elevator followed by smoothening of sharp bony edges with a bone file. Intraoperative digital RVG was taken to ensure the complete removal of radiopaque masses. Sutures were then placed [Figure 3] and the specimen [Figure 4] was sent for histopathological examination for confirmatory diagnosis.

\section{Histopathological findings}

$\mathrm{H}$ and $\mathrm{E}$ stained decalcified section showed the presence of eosinophilic tubular structure

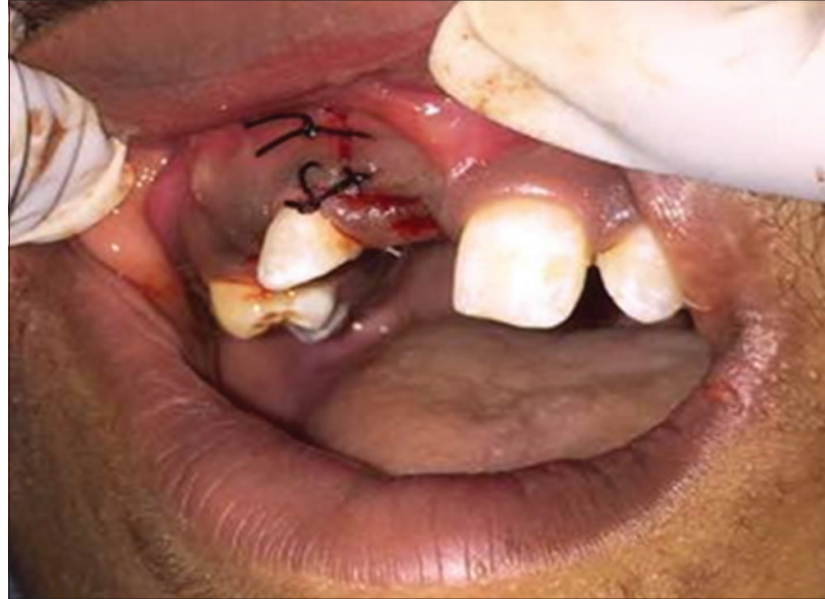

Figure 3: Clinical picture showing placement of sutures

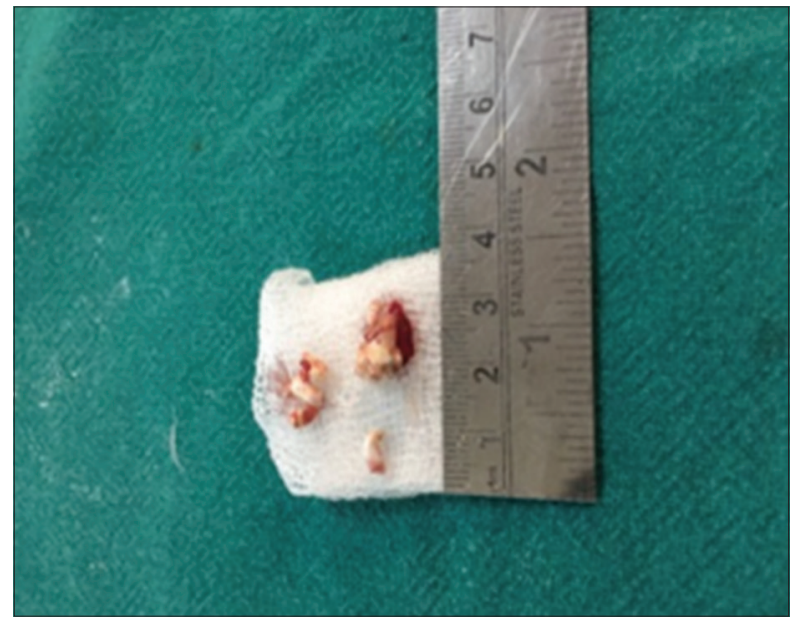

Figure 4: Clinical picture showing the specimen that was sent for histopathological examination for confirmatory diagnosis

resembling dentin surrounding the pulpal space interspersed with dense collagen fibers, endotheliallined blood vessels, and few inflammatory cells chiefly composed of lymphocytes. At few areas, hematoxyphilic fibrillar enamel matrix-like material is evident. $\mathrm{H}$ and $\mathrm{E}$ stained section of the soft tissue shows the presence of dense fibrous connective tissue interspersed with inflammatory cells in some areas; these features are suggestive of compound odontoma [Figure 5].

The patient was recalled after 1 week and 1 month after the procedure which showed complete healing [Figure 6].

\section{DISCUSSION}

Odontoma is the most common type of odontogenic tumor or hamartoma (not a true 


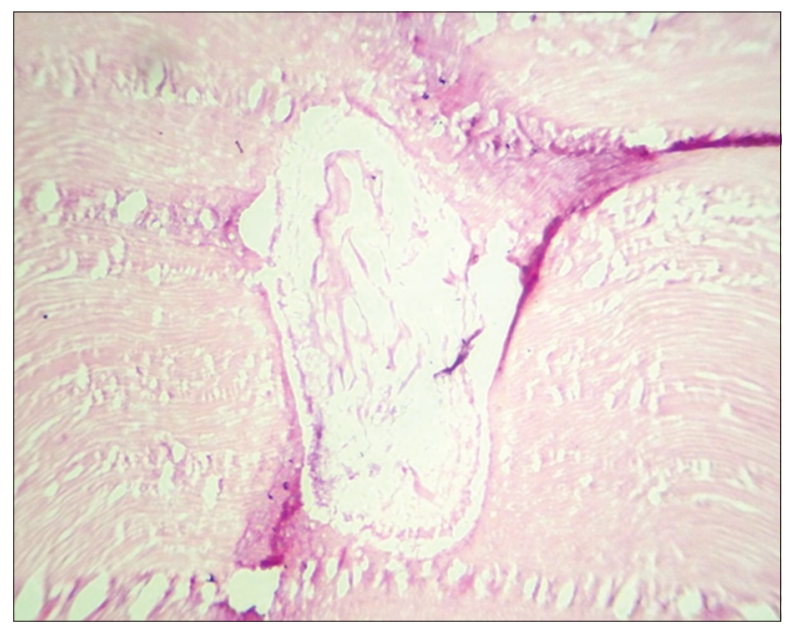

Figure 5: $\mathrm{H}$ and $\mathrm{E}$ stained decalcified section shows the presence of eosinophilic tubular structure resembling dentin surrounding the pulpal space interspersed with dense collagen fibers, endothelial lined blood vessels, and few inflammatory cells chiefly composed of lymphocytes. These features are suggestive of compound odontoma

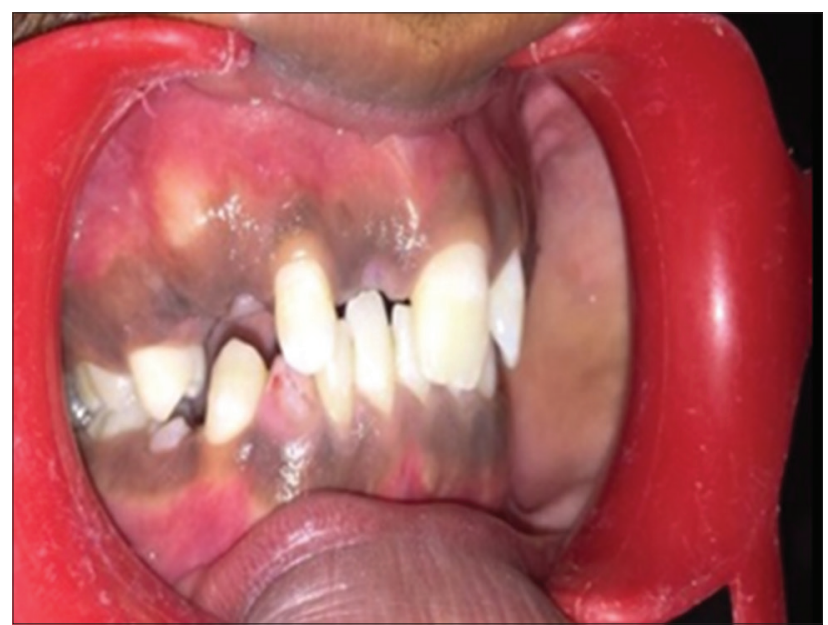

Figure 6: Post-operative picture showing complete healing after 1 month

tumor). ${ }^{[5]}$ Hitchin suggested that odontomas inherited through a mutant gene or interference, possibly postnatal, with genetic control of tooth development. In humans, there is a tendency for the lamina between the tooth germs to disintegrate into clumps of cells. The persistence of the lamina may be an important factor in the etiology of complex or compound odontomas, and either of these may occur instead of a tooth. ${ }^{[5]}$

Odontomas have been associated with trauma during primary dentition, inflammatory and infectious processes, hereditary anomalies
(Gardner syndrome and Hermann syndrome), and odontoblasts hyperactivity, and alterations in the genetic components are responsible for controlling dental development. ${ }^{[5]}$

According to the WHO classification (2005), two types of odontomas can be found: Complex odontomas and compound odontomas. In complex odontomas, dental tissues are arranged in a disorderly pattern. In compound odontomas, they are arranged more orderly. Complex odontomas are less common than the compound odontoma in the ratio of $1: 27{ }^{[6]}$

\section{follows:}

Gravey et al. classified compound odontomas as

1. Denticulo type: Composed of two or more separated denticles having crown and root, dental hard tissue resembling that of the tooth.

2. Particulate type: Composed of two or more separate masses or particles, bearing no resemblance to the tooth.

3. Denticulo-particulate type: In this, both denticles and particles are present together. ${ }^{[2]}$

About $70 \%$ of the unerupted tooth have been reported to erupt spontaneously after removal of obstruction. ${ }^{[7]}$ The same was seen in the present case also.

Kaban reported that odontomas are easily enucleated and adjacent teeth that may have been displaced by the lesion are seldom harmed by the excision because they are usually separated from the lesion by a septum of bone. ${ }^{\left[{ }^{[8]}\right.}$ The early diagnosis of odontomas will reduce the chance of the development of malocclusion and pathological changes in that region. ${ }^{[9]}$

Some authors recommended the exposure of the unerupted tooth at the time of surgery and placement of bonded attachment and ligature/echain for orthodontic traction, to facilitate rapid eruption. This approach may result in a poor gingival margin, inadequate gingival tissue attachment, and a discrepancy of the gingival level between the exposed tooth and its neighboring teeth. ${ }^{[9]}$ In the present case, only one-third of root formation was completed, so left undisturbed for the normal physiologic eruption of the tooth.

\section{REFERENCES}

1. Serra-Serra G, Berini-Aytés L, Gay-Escoda C. Erupted odontomas: A report of three cases and review of the literature. Med Oral Patol Oral Cir Bucal 2009;14:299-303.

2. Satish V, Prabhadevi MC, Sharma R. Odontome: A brief overview. Int J Clin Pediatr Dent 2011;4:177-85. 
3. Rajendran R, Sivapathsundaram B. Shafer's Textbook of Oral Pathology. $5^{\text {th }}$ ed. Amsterdam: Elsevier; 2006. p. 405-6.

4. Reddy GS, Reddy GV, Sidhartha B, Sriharsha K, Koshy J, Sultana R, et al. Large complex odontoma of mandible in a young boy: A rare and unusual case report. Case Rep Dent 2014;2014:854986.

5. Mehta D, Raval N, Udhani S, Parekh V, Modi C. An unusual case report of erupted odontoma. Case Rep Dent 2013;2013:570954.

6. Vengal M, Arora H, Ghosh S, Pai KM. Large erupting complex odontoma: A case report. J Can Dent Assoc
2007;73:169-73.

7. Tanki JZ, Naqash TA, Gupta A, Singh R, Jamwal A. Impacted maxillary incisors: Causes diagnosis and management. J Dent Med Sci 2013;5:41-5.

8. Qazia HS, Ibrahim MW, Siddiqi KM, Zia AU. Compound odontoma causing impaction of mandibular canine: A case report. Pak Orthod J 2011;3:25-31.

9. Maden EA, Altun C, Sencimen M. A case report of compound odontoma associated with an unerupted maxillary central incisor. Arch Clin Exp Surg 2014;3:47-51. 